\title{
Se former dans l'enseignement supérieur en coanimant : une experience entre biologistes et psychopédagogues
}

\author{
Hervé Duchauffour ${ }^{\mathrm{a}}$
}

CERLIS-Université Paris-Descartes/ESPE de l'académie de Paris- Université Paris-Sorbonne, France

\begin{abstract}
Résumé. Cet article expose en quoi une coanimation entre enseignants des sciences humaines et enseignants biologistes a pu conduire ces derniers à modifier leurs représentations et leurs pratiques d'enseignement dans leur discipline, à partir d'une expérience vécue dans le cadre de modules de préprofessionnalisation.
\end{abstract}

\begin{abstract}
This article describes how a co-teaching by human sciences professors and biologist professors could lead the latter to rethink their teaching personae and, for some, to modify their current teaching practices, from an experience lived in the implementation of pre-professionalization modules.
\end{abstract}

\section{La preprofessionnalisation, un double enjeu de formation?}

\subsection{Un contexte : le « Plan Réussite en Licence » (PRL)}

C'est dans le cadre de la mise en place du «Plan réussite en licence $»^{1}$ au sein de l'université ParisDiderot que cette étude a vu le jour. Le département Sciences de la nature et de la vie a inscrit une série de modules déployés autour de la préprofessionnalisation (PP) dans sa maquette de la licence ${ }^{2}$ pour lutter contre l'échec important des étudiants de première année (Erlich, Verley, 2010, 82) et pour permettre aux étudiants brillants dans leur discipline de mieux réussir leurs entretiens de recrutement en master ou pour leurs recherches de stage ${ }^{3}$. Il s'agit de les accompagner dans la construction de

\footnotetext{
a e-mail : herve.duchauffour@paris-sorbonne.fr

${ }^{1}$ Plan annoncé par la ministre de l'enseignement supérieur et de la recherche Valérie Pécresse en décembre 2007.

2 Maquette 2009-2013. Environ 300 étudiants sont concernés par ces unités de formation.

${ }^{3}$ Les enseignants de licence ont effectivement noté que certains étudiants brillants en cours ne parvenaient pas à convaincre les commissions lors de recrutement pour l'inscription dans des masters sélectifs.
}

This is an Open Access article distributed under the terms of the Creative Commons Attribution License 4.0, which permits unrestricted use, distribution, and reproduction in any medium, provided the original work is properly cited. 
leur projet professionnel tout au long de la licence en guidant leur orientation avec une démarche appropriée (Vasconcellos, 2006). C'est l'élaboration de cette "démarche » qui a engendré une large réflexion sur les méthodes pédagogiques des enseignants participant à ces actions. C'est cette mise en question des modes d'enseignement grâce aux actions de préprofessionnalisation qui fait l'objet de cet article.

\subsection{La rencontre des enseignants des sciences du vivant et des sciences humaines}

Le montage de ces modules s'est opéré avec la volonté de sortir les contenus d'un enfermement disciplinaire. Cela a eu pour premier effet de bousculer l'équipe enseignante qui a vite repéré un danger potentiel dans cette situation : celui de perdre la maîtrise de son enseignement et donc de fragiliser sa stature de «spécialistes disciplinaires ». En effet, la figure du bon enseignant dans l'enseignement supérieur repose sur l'expertise dans son domaine de recherche et s'inscrit dans la logique d'une allégeance à sa discipline contribuant ainsi à définir l'automaticité : bon chercheur égal bon enseignant (Rege Colet, Berthiaume, 2009, 138). Cette fragilité mise au jour, la responsable du département $\mathrm{SNV}^{4}$ a dû convaincre ses collègues pour qu'ils surmontent leurs réticences et acceptent de participer à cette entreprise vécue à risques.

C'est en choisissant d'associer des collègues issus des sciences humaines (enseignants en sciences de l'éducation, formateurs en $\mathrm{IUFM}^{5}$, responsable d'orientation) pour élaborer les contenus de séances que l'obstacle a été levé. Formateurs des sciences humaines et enseignants biologistes se sont ainsi constitués en binômes pour encadrer les groupes. Sur les trois années d'expérience, ces binômes ont varié en fonction des disponibilités horaires des uns et des autres, en respectant la parité sciences humaines/sciences « dures ». Ainsi, des relations se sont nouées à la fois lors des séances plénières mais aussi de manière plus individuelle dans des échanges au sein des binômes, avant et après les séances de travail avec les étudiants.

L'intérêt de cette mixité repose sur deux axes. Le premier est lié aux objectifs de ces formations. Le cœur des contenus travaillés dans les diverses unités d'enseignement renvoie à des notions et concepts de la communication, de l'argumentation et à ceux de la professionnalisation comme le rôle, le statut, le métier. Ces contenus, assez éloignés du champ de la biologie -restée en toile de fond- devenaient objets d'apprentissage pour les étudiants mais aussi en partie pour les enseignants biologistes. Le second renvoie aux méthodes d'apprentissage utilisées en formation et dans les champs de la pédagogie et de la psychologie sociale. Celles-ci réfèrent à des démarches socioconstructivistes assez éloignées d'un enseignement transmissif plutôt habituel dans les amphithéâtres de l'université. Ces démarches qui placent l'apprenant au centre d'un dispositif ont pour but de le rendre pleinement acteur de ses apprentissages et d'impliquer le groupe de pairs dans la construction des savoirs (Bruner, 1966). Ainsi à chaque séance, les modalités de travail des étudiants ont varié conduisant les enseignants à des modes d'interaction tout aussi variés. Là encore, il s'est agi d'un apprentissage pour certains enseignants.

Attendus sur ces registres, les enseignants des sciences humaines ont dû jouer le rôle de garant dans la mise en œuvre des actions de formation auprès des étudiants. C'est leur expertise dans le domaine spécifique de la psychopédagogie ${ }^{6}$ en regard des compétences déployées par les enseignants scientifiques dans leur propre domaine qui a été mise à contribution.

\footnotetext{
${ }^{4}$ Département sous la direction du professeure Claude Bazin, sans qui par ailleurs cette étude n'aurait pu avoir lieu. Qu'elle en soit très sincèrement remerciée.

5 Institut universitaire de formation des maitres devenu ESPE, École supérieure du professorat et de l'éducation.

${ }^{6}$ Ce sont les enseignants biologistes qui ont « réuni » les champs d'intervention des enseignants non biologistes sous le vocable «psychopédagogie » et les ont qualifiés de «psychopédagogues ». Elle qualifie leur activité d'enseignants attentifs aux modes de construction des savoirs du sujet en s'appuyant sur les apports de la psychologie.
} 


\subsection{Des modules de formation coanimés}

Les enseignants biologistes en participant à ce travail ont découvert que le métier d'étudiant ne va pas de soi. Celui-ci commence à se forger en référence au métier d'élève. Chaque individu « habite son métier à sa manière » en fonction de l'environnement avec lequel il interagit (Perrenoud, 2000, 172) et ce métier devient aujourd'hui objet d'apprentissage. Ainsi, les modules obligatoires de préprofessionnalisation conduisent les étudiants à apprendre à faire des choix pertinents que ce soit pour la poursuite de leurs études, dans le cadre d'un stage ou de l'entrée dans la vie active. Ceux-ci s'accompagnent d'une réflexion sur la posture à adopter en fonction de la situation. Pour cela, les actions de formation prennent la forme d'atelier d'échanges, de recherches documentaires, de jeux de rôle ou d'exposés. Une autre unité d'enseignement, transversale et optionnelle, « Regards critiques, argumentation et communication »s'adresse à tous les étudiants de licence quelle que soit leur discipline. Celle-ci a pour objectif d'éprouver et de mettre en valeur les compétences des étudiants dans le registre de la communication et de l'argumentation.

Ainsi, la démarche pédagogique à l'œuvre dans cet enseignement réfère plus à de l'accompagnement (Paul, 2004, 149) qu'à une démarche d'enseignement classique, plus transmissive. Il s'agit pour les enseignants de poser un cadre de travail mais d'être ensuite très en retrait. Les étudiants sont très mobilisés à la fois pour travailler les contenus en jeu mais aussi pour les faire vivre pendant la séance de cours. Cette modalité de travail permet une centration sur le sujet apprenant plus que sur la transmission de savoirs académiques, ceux-ci étant malgré tout le support obligé de cette démarche de travail. Ce sont ces modalités de travail qui ont ébranlé plus ou moins fortement les représentations que se font les enseignants de leur métier.

\section{Problématique et méthode}

\subsection{Interroger la coanimation}

Puisque que les pratiques enseignantes jouent un rôle déterminant dans la réussite des étudiants dans le cycle de licence de l'université où l'effet «maître » prend tout son sens (Attali et Bressoux, 2002), il nous a semblé intéressant d'analyser le dispositif mis en place dans le cadre de la préprofessionnalisation en focalisant notre travail sur les effets de la coanimation. Coanimation qui repose sur « le respect et la mise en œuvre des initiatives planifiées conjointement »(Carnus, 2010, 36). Ce focus est le fruit d'un constat : au regard des propos tenus lors des réunions préparatoires, a priori, les biologistes ne se sentaient pas en mesure d'animer seuls ce type de module, d'où un certain nombre de questions.

Pourquoi ces enseignants avaient-ils accepté cette participation ? Relevait-elle d'un besoin ou d'un désir de faire évoluer leur professionnalité au regard des nouveaux enjeux posés aux universités (Leclercq, 2009, 233) ? S'agissait-il de relever un défi, parmi d'autres dont l'institution se préoccupe peu tant l'entrée en fonction d'enseignant-chercheur se fait sous le coup de l'excellence de la recherche (Langevin et Bruneau, 2000, 8) ?

En quoi cette coanimation entraînerait-elle des modifications à la fois sur le plan des représentations de l'activité enseignante mais aussi des modifications sur le plan des pratiques (Trigwell et Prosser, 2004 ; Langevin, Bilodeau, Boisclair et Bracco, 2007). ? En quoi la coanimation pouvait-elle participer à cette évolution et quel impact pouvait-elle avoir (Kirkpatrick, 1994) ? Entre autres, y aurait-il des changements du point de vue des connaissances, des compétences, des attitudes, des comportements des scientifiques dans leurs enseignements habituels (Stes et Van Petegem, 2011,16)?

Il s'agissait aussi de vérifier si l'affirmation : «c'est en enseignant qu'on apprend à enseigner » (Knight, Tait, Yorke, 2006) était dans ce cas opératoire, sachant qu'ici, les contenus à enseigner n'étaient pas du domaine de la biologie. Et que par ailleurs, la posture à construire relevait plus d'articulation entre modèle d'enseignant transmetteur et celui d'accompagnateur de développement personnel (Barbier, 2005). 


\subsection{La professionnalité de l'enseignant du supérieur}

Inscrite dans le champ de la sociologie des professions, cette étude rend compte de la manière dont la construction d'une identité professionnelle est un long processus (Wittorski, 2007). Celui-ci incorpore la manière dont on s'identifie à ses pairs, la maitrise de compétences spécifiques, la représentation qu'on a de son métier et l'image qui nous est renvoyée par notre environnement. Mais elle questionne aussi la spécificité d'une formation d'adulte qui se caractérise par une lutte contre l'obsolescence (Léon, 1971, 102-103). Le dispositif mis en place à destination des étudiants a aussi répondu à la volonté des enseignants scientifiques de se mettre à l'épreuve dans des situations de cours peu habituelles, renvoyant ainsi le dispositif au paradigme non énoncé mais bien présent de l'apprentissage informel (Carré et Charbonnier, 2003). Les temps de régulation qui ont permis de faire émerger nombre d'éléments pris en compte dans notre analyse, s'ils n'en avaient pas la rigueur formelle et le cadre spécifique, pouvaient s'inscrire dans le champ des analyses de pratiques professionnelles (Blanchard-Laville, Fablet, 1998).

\subsection{Une approche qualitative}

Les résultats de cette étude reposent sur l'analyse d'un corpus constitué de questionnaires ${ }^{7}$ renseignés par la dizaine d'enseignants-chercheurs biologistes ${ }^{8}$ qui participent aux coanimations. Une partie du questionnaire renvoie au profil des répondants : ancienneté dans la fonction d'enseignant-chercheur, niveaux d'enseignement, conditions d'entrée dans la fonction, modules de préprofessionnalisation sur lesquels les interventions portent et ancienneté dans la participation à ces actions. Une deuxième partie interroge les contextes d'enseignement en dehors de ces modules, à savoir : Travaux Pratiques (TP), Travaux Dirigés (TD) ou Cours Magistraux et les préférences affichées ainsi que les motifs invoqués. Une troisième partie questionne le rapport à l'enseignement en préprofessionnalisation et vise à dire en quoi celui-ci est vécu comme différent de l'enseignement habituel et à caractériser les modes d'intervention. Cette partie expose aussi comment l'enseignant a pu modifier ses représentations du métier d'enseignant dans le supérieur, voire modifier sa pratique. D'autre part, l'analyse du verbatim constitué lors des réunions de régulation contribue à préciser les changements opérés ou non. Ces réunions organisées à chaque fin d'année ${ }^{9}$ ont pour objet de revenir sur l'expérience vécue et d'en analyser le déroulement. In fine, l'évaluation faite par les enseignants de ces modules destinés aux étudiants rend compte du regard qu'ils portent sur leur propre vécu d'animateur de ces groupes. Lors de ces réunions sont présents tous les enseignants participant à ces enseignements.

Les analyses tirées de ce corpus restreint doivent être considérées comme l'expression d'une réalité et toute précaution est à prendre pour en faire une généralisation. La méthode d'analyse se réfère globalement aux analyses de contenu mais plus précisément du discours qui tient compte du lieu d'énonciation (Maingueneau, 1996). Les réponses aux questions ouvertes ont ainsi été catégorisées en fonction des thématiques abordées et traitées pour « faire advenir un sens qui n'était pas visible jusque là, sans qu'il soit pour autant intentionnellement caché » (Robert Bouillaguet, 2002, p. 55). L'interprétation tient compte à la fois des hypothèses, du locuteur et du contexte d'élocution. Les réponses aux questions fermées ont été croisées avec l'interprétation faite des questions ouvertes autour d'hypothèses comme l'ancienneté joue-t-elle un rôle dans la manière d'appréhender ces nouvelles formations ? Ou encore, la pratique habituelle du format TP ou TD a-t-elle été un facteur d'adhésion à celles-ci ? Comme à aucun moment nous nous sommes trouvés dans la situation d'observer les collègues dans leur enseignement disciplinaire, seuls leurs dires, écrits ou parlés, ont nourri cette analyse.

\footnotetext{
7 Quinze questions : huit questions fermées, sept questions ouvertes et une rubrique finale « commentaire ».

8 Dix enseignants sur les douze qui participent ont répondu au questionnaire.

${ }^{9}$ Ont été retenues les réunions de régulation de juin 2011, juin 2012 et juin 2013.
} 
Les sciences humaines dans les parcours scientifiques et techniques professionnalisants

\section{Relever un défi}

\subsection{Des enseignants motivés}

Les enseignants engagés volontairement dans cette expérience ont une ancienneté de trois à trente ans, la moyenne étant de douze ans sept mois. Ce sont majoritairement des femmes, seuls deux hommes ont participé à ce travail. Par ailleurs, mis à part un professeur des universités, ils ont tous le grade de maître de conférences. Six enseignants participent à ces actions depuis leur création et leur mise en œuvre, quatre autres ont intégré le dispositif en deuxième année. Un seul enseigne régulièrement en amphithéâtre, les autres majoritairement en TP ou TD et préfèrent enseigner en TP.

Les premières analyses rendent compte de la motivation des enseignants à participer à ce type d'enseignement. C'est une véritable aventure dans laquelle ils se sont lancés tant contenus et méthodes en jeu étaient éloignés de leur activité quotidienne. En cela, ils démontrent que l'université tend aussi à lutter contre l'immobilisme dans lequel on voudrait l'enfermer (Rege Colet, Berthiaume, 2009, 140). Divers motifs fondent cet engagement :

- La forme du cours, les ateliers de préprofessionnalisation ressemblent à un TP ou TD, cadres dans lesquels ils enseignent habituellement. Cette similitude apparente de la forme a donc été attrayante, entre autres parce qu'elle est pour eux synonyme de proximité avec les étudiants ${ }^{10}$. Pour eux, ce lien favorisé permet de mieux appréhender l'activité intellectuelle des étudiants ainsi que leur démarche pour résoudre les situations problèmes qui leur sont posées : "on est plus enclin à solliciter les étudiants pour qu'ils trouvent eux-mêmes dans leurs propres capacités des réponses... ${ }^{11}$.

- La capacité à prendre de la distance par rapport à des pratiques d'enseignement purement liées à la discipline et cela quelle que soit leur ancienneté. Ils imaginaient leur intervention possible en PP puisque cette mise à distance était en quelque sorte étayée par la présence d'un collègue enseignant non scientifique : "voir quelqu'un d'autre enseigner et gérer un groupe m'a beaucoup aidé » ${ }^{12}$.

- Le besoin de se rassurer, une enseignante, la seule dont les pratiques habituelles diffèrent peu de celles préconisées en PP avait « remarqué que sa façon d'enseigner était différente des autres ». Elle n'avait pas trouvé « de lieu où échanger » aussi elle cherchait à être "moins seule » ${ }^{13}$ confirmant la nécessité et le rôle pédagogique que peuvent avoir les échanges entre collègues (Cuisinier, Jeoffrion et Schneider, 2006, 242-243).

On peut conclure sur ce point en précisant qu'aucun enseignant engagé dans ces modules n'a quitté l'expérience et que leur engagement reste entier.

\subsection{Une identité professionnelle questionnée}

Si la motivation est restée intacte, il faut quand même souligner que les commentaires libres laissés en fin de questionnaire montrent un écart réel entre ce que les participants avaient imaginé et la réalité de l'expérience. Les représentations ont été bouleversées pour la majorité d'entre eux (neuf sur dix) : c'est une nouvelle conception de l'enseignement qui leur est apparue. L'analyse des réponses rend compte de cet écart. Elle permet de relater la manière dont ils se perçoivent dans cette activité d'enseignement. C'est leur identité professionnelle qui a été en quelque sorte défiée, celle-ci s'étant construite sur le schéma frontal classique de l'enseignant transmetteur de savoirs. Ils ne se perçoivent pas ici comme enseignants mais plutôt majoritairement comme " guide, incitateur, accompagnateur ». Très à la marge, les termes de « révélateur, encadrant» ont été employés. Pour l'un d'entre eux, n'ayant que trois années d'expérience dans le métier, le propos est plus catégorique : "on anime plus que l'on enseigne ».

\footnotetext{
10 Les groupes étaient constitués de 12 à 30 étudiants.

11 Enseignante de licence, 17 ans d'ancienneté.

12 Enseignante de licence et master, 4 ans d'ancienneté.

13 Enseignante de licence et master, 21 ans d'ancienneté.
} 
Ces termes trouvent une explication dans la manière dont ils parlent de cette activité. Activité assez éloignée pour eux d'une situation d'apprentissage " par transmission de savoirs » puisqu'il n'y a pas de programme à proprement parler et que les contenus se construisent au fur et à mesure des séances, créant en cela une proximité avec l'« apprentissage par tutorat » (Pastré, 2008, 65) où enseignant et enseignés se découvrent mutuellement au cours des séances. Les objectifs d'apprentissage d'ailleurs ne sont pas « disciplinaires » ou du moins ne relèvent pas de leur discipline d'enseignement, mais mettent en jeu des compétences transversales comme : savoir se présenter, s'exprimer dans différents contextes, argumenter, utiliser des outils de recherche, analyser, organiser et traiter des informations, s'exposer, être à l'écoute, rédiger. Il est donc difficile pour eux de définir ce qui se travaille.

D'autre part, l'activité enseignante est très différente de celle pratiquée quotidiennement : il s'agit beaucoup plus de "se taire et écouter » que de «faire cours ». L'enseignant ne se sent pas dans une position de savoir car il n'y a pas de réponse unique attendue aux situations problèmes posées. Le décalage peut être possible et il est accepté entre la réponse imaginée et la réponse donnée. Cet écart est une grande nouveauté puisqu'habituellement tous préparent leurs cours et savent à quoi s'attendre en termes d'expérimentation et de résultats. Ainsi, c'est parfois la frustration par rapport à certaines attentes qui se fait jour.

Autre motif explicatif concernant ces interrogations, l'enseignant se trouve plus dans une position de solliciter que d'expliquer et ce dispositif fondé sur l'échange avec les étudiants ne prête pas au "forcing ». Ce terme employé par certains biologistes renvoie à la pression qu'ils ressentent dans l'application du programme dans un temps donné ainsi que l'urgence qu'il y a à mener à bien certaines expériences dans leur domaine d'exercice. Ici, c'est au rythme de l'étudiant que les apprentissages se font dans la contrainte du respect du calendrier fixé.

Les questions autour de la professionnalité de l'enseignant du supérieur dans ce contexte ont pu ainsi être posées, principalement autour de la relation à l'étudiant dans la construction des savoirs. Les motifs de ces interrogations ont été identifiés. Mais, il reste à démontrer quels effets marquants cela peut avoir dans le temps en termes de représentation et de pratiques.

\section{Des effets réels mais limités}

\subsection{Des représentations modifiées : le métier d'enseignant vu sous un jour nouveau}

Sept professeurs sur dix ont déclaré porter un regard différent sur leur métier d'enseignant. Concernant les trois enseignants dont les représentations n'auraient pas évolué, l'analyse conduit à identifier des éléments explicatifs. Pour l'un d'entre eux, cette participation allait de soi puisque déjà enclin dans sa pratique professionnelle à s'ouvrir à des méthodes pédagogiques prônant les interactions ${ }^{14}$. Pour un autre, la représentation du métier d'enseignant s'ancrait déjà dans un éventail de possibles allant du cours magistral -lié à sa pratique- à la participation active des apprenants ${ }^{15}$. Pour la troisième personne, le professeur reste celui qui enseigne et transmet un savoir sur un mode magistral. C'est un enseignant qui aime les cours en amphithéâtre et déclare sa passion pour sa discipline ${ }^{16}$.

L'analyse du corpus montre que plusieurs éléments fondent le changement de représentation du métier et que c'est grâce au dispositif de coanimation que cela a été rendu possible : "l'interaction avec nos collègues psychopédagogues m'apporte une nouvelle vision de l'enseignement où l'étudiant construit son savoir de façon plus active ${ }^{17}$.

\footnotetext{
14 Il s'agit de l'enseignante ayant toujours enseigné « différemment », 21 ans d'ancienneté.

15 Cette ouverture se serait construite à l'aune des échanges réguliers que cet enseignant a avec son conjoint professeur des écoles. Il a 3 ans d'ancienneté.

16 Enseignante de licence et master, 15 ans d'ancienneté, dissocie ce «type d'enseignement » de l'enseignement.

${ }^{17}$ Enseignante, 7 ans d'ancienneté, intervient en licence et en master principalement, sous forme de TD.
} 
C'est donc le dispositif lui-même qui conduit les enseignants à travailler sous le regard de leurs collègues qui permet de faire apparaître les différences. C'est la posture " être parmi les étudiants...pour les guider » qui est manifestement l'élément le plus sensible. L'intervention non directive, " analyseur » plus que prescripteur, "récepteur » plus qu'émetteur, autant de figures qui ont semblé marquer les esprits des enseignants scientifiques. Tout comme les contenus qui pour certains d'entre eux paraissaient très éloignés d'un savoir académique. Dans ce dispositif, les «psychopédagogues » ont fait apparaitre des modalités d'enseignement singulières qui ont conduit à interroger même la fonction d'enseignement. Que faisions-nous ? En quoi cela pouvait-il être assimilé à de l'enseignement ? C'est en revenant sur les objectifs de la préprofessionnalisation que les réponses furent apportées ; entre autres, en déclinant les compétences mises au travail pendant les séances.

Le deuxième élément troublant les représentations renvoie aux images que les scientifiques avaient des étudiants. La majorité d'entre eux les enfermaient dans un rôle de "faiseur » répondant à une commande. Les modalités de travail qui conduisent les étudiants à se prendre en charge, à s'exposer, ont permis de mettre en évidence les capacités de ceux-ci à être autonomes par rapport à une commande passée par l'enseignant. Tout comme de pouvoir répondre à celle-ci en empruntant des chemins très variés. Ce que résume une enseignante : "Nous sommes moins dans une position de savoir avec une réponse unique comme cela peut-être le cas pour un TD ou un cours magistral de sciences ${ }^{18}$. La forme des interventions, un équilibre important entre écrit et oral pour " Regards critiques », une forte implication par la parole dans les modules de PP, ont mis en évidence que le sacrosaint polycopié des salles de sciences pouvait être remplacé sans nuire à l'apprentissage. La découverte de l'autonomie des étudiants renvoie de manière corolaire à la toute puissance bouleversée de l'enseignant. Il s'agitlà d'une remise en question fondamentale de la stature du professionnel qui sait, face à celui qui ne sait pas. Remise en question déjà avancée avec les nouvelles technologies de l'information et de la communication (NTIC), qui permettent aux étudiants de construire certains savoirs en dehors de l'enseignant et voire même de vérifier si ce que l'enseignant dit est juste.

Un troisième élément mis au jour rend compte aussi de ce qui a conduit aux changements. Dans ces dispositifs, les étudiants sont vus dans leur globalité et pas seulement comme des apprenants biologistes : "on rencontre les étudiants comme des individualités...on les voit se construire au long des séances... on les voit à leur avantage... leurs qualités humaines...leur créativité " ${ }^{19}$. Les situations telles que devoir présenter une œuvre de son choix dans le champ de son choix, ou encore participer à un débat ou faire un poster dont on aura choisi la thématique, sont autant de moments où l'étudiant peut révéler sa personnalité et faire découvrir ses capacités sous un autre jour. La prise en compte de la dimension psychologique, sans que l'enseignement ne soit psychologisant, a été un élément déstabilisateur des représentations. En quoi, l'enseignant doit-il s'intéresser un minimum à la psychologie de ses élèves, a été une question qui a fait débat au niveau de l'équipe.

Un quatrième élément a vite émergé. Pour certains il a été à la fois une découverte et une réelle difficulté, c'est de vivre le passage de l'enseignant qui parle, "beaucoup », dira un scientifique, à celui qui écoute. L'obstacle passé, une autre difficulté est apparue : que faire de ce qui est entendu ? Là encore, le dispositif de coanimation a permis de réguler cette situation, les psychopédagogues étant les plus à même de faire face à l'inattendu de certaines situations, remarques ou propositions ne relevant pas du domaine des sciences du vivant. "Travailler à deux sécurise beaucoup sur des erreurs d'interprétation que nous pourrions commettre ${ }^{20}$ a précisé une biologiste.

\footnotetext{
18 Enseignante, 6 ans d'ancienneté, préfère le TP comme forme d'enseignement.

${ }^{19}$ Enseignante, 30 ans d'ancienneté, intervient majoritairement en amphithéâtre.

${ }^{20}$ Cf. note 19 .
} 


\subsection{Sur le plan des pratiques}

A ce stade de l'expérience, après trois années de participation pour la majorité d'entre eux, la moitié déclare avoir effectivement modifié sa pratique face aux étudiants dans les cours qu'ils ont l'habitude d'assurer. En cela, on peut dire que le travail de régulation venu en appui de la mise en place des modules a permis de dépasser la simple « prise de conscience » pour conduire à la modification des schèmes de formation (Perrenoud, 2001, 195).

Les principales évolutions relevées par ceux qui ont pu en faire le constat renvoient à la place donnée aux étudiants pendant les cours : «je suis plus proche et plus à l'écoute des étudiants " ${ }^{21}$. C'est la personne de l'étudiant qui est convoquée et pas simplement l'apprenant, scientifique en herbe : "j'essaie d'être à l'écoute de leurs préoccupations sur leur avenir en général et sur leur ressenti des enseignements qu'ils suivent, sans pour autant tout prendre pour argent comptant $»^{22}$. C'est dans la logique de cette invitation nouvelle à être soi dans sa globalité, une invitation à participer davantage. Ainsi, les cours sont plus interactifs. L'interaction ne se situe pas spécifiquement entre l'enseignant et l'étudiant mais les échanges entre étudiants sont favorisés autour de dispositif de travail en commun : "plus d'échanges, de travail de réflexion voire de silence pour forcer les étudiants à s'impliquer ${ }^{23}$. Entre autres, un enseignant a narré la manière dont il avait modifié ses TD laissant incrédules les étudiants auxquels ils s'adressaient, tant le formatage académique semble être déjà établi dès les premiers mois universitaires. Bousculer ce qui semble être attendu - je suis étudiant donc je me tais - a été un vrai défi déstabilisant pour les deux parties.

Une autre transformation majeure dans la pratique enseignante a été de s'imposer de la retenue dans la parole : «je laisse parler les étudiants plus souvent et plus longtemps... pour ne pas bloquer leur créativité $»^{24}$. Être plus à l'écoute est sans aucun doute l'élément le plus significatif de ces évolutions mais aussi le plus difficile à faire vivre pour sept des dix enseignants interrogés. Avec pour corolaire de s'armer de patience pour attendre que les réponses viennent des étudiants sans induire celles-ci.

\section{Conclusion}

L'expérience de la coanimation est devenue un outil de formation ou d'autoformation. Elle a conduit les enseignants-chercheurs des disciplines des sciences de la nature et de la vie à construire de nouvelles représentations du métier d'enseignant. Certains sont même allés jusqu'à modifier leur action pédagogique pourtant placée sous le contrôle d'un habitus fort en sortant des routines « qui n'exigent plus la mobilisation explicite de savoirs et de règles » (Perrenoud, 2001, 184). Ils se sont efforcés de proposer à leurs étudiants de nouvelles formes de réflexion. Ils ont fait appel aux interactions, entre autres à partir d'un travail de groupe et ont pris pour eux-mêmes des risques épousant ainsi la figure de l'enseignant «stratège et entraineur » (Langevin, Bruneau, 2000, 16). Ces effets de style ont contribué dans le même temps à « déstabiliser » les étudiants très surpris par ces normes fluctuantes (Monfort, 2000, 66) contraignant dans un effet boomerang l'enseignant à repenser le contrat didactique.

Cet accompagnement professionnel « caché » que nous pourrions définir comme une pratique de régulation et non pas de conformisation a pris forme d'étayage (Vial, Caparros-Mencacci, 2007, 76) conduisant chacun à « faire évoluer son dispositif de représentation mentale, à connaître ses moteurs, ses freins et ses limites » (Forestier, 2002, 15). «Se questionner soi-même » comme le dit une participante. Les limites majeures identifiées renvoient plus à la difficulté à contrôler la place de sa parole, sa capacité à ne pas induire ou donner les réponses, ou encore à développer son aptitude à écouter. La peur de

\footnotetext{
21 Cf. note 17.

22 Enseignante, 14 ans d'ancienneté, intervenant principalement en TP et TD et préférant les TP.

23 Cf. note 18 .

24 Cf. note 12 .
} 
« retomber très vite dans la transmission classique des connaissances » est évoquée. Cependant, le moteur puissant est l'envie générale de progresser sur cet autre chemin.

Cet étayage, présenté comme nécessaire par les participants, montre cependant ses limites puisque les enseignants les plus sensibles au changement de posture ont formulé de manière explicite un besoin de formation. C'est une demande d'aide pour aller plus loin encore dans leur évolution personnelle, rappelant d'une certaine manière que la formation initiale d'un enseignant chercheur est bien une formation à la recherche et pas une formation à enseigner (Fave-Bonnet, 2011, 135). Lors des réunions de régulation, ce qui est apparu comme étant le plus déstabilisant c'est de trouver sa place au sein du triangle pédagogique : Savoir-Enseignant-Apprenant (Houssaye, 2000) réactualisant ainsi la question de la formation des universitaires (Romainville, Donnay, 1996).

La nécessité de passer par la compétence s'est faite jour pour démontrer que ces modules avaient effectivement eu pour effet que les étudiants apprennent quelque chose (Leclercq, 2012, 168) et cela grâce à l'action des enseignants. Ces savoirs basés sur des contenus disciplinaires ne relevant pas des sciences de la nature et du vivant ont d'abord dû trouver écho chez les enseignants biologistes pour qu'ils se sentent aptes à les faire travailler.

Ces compétences ayant été formalisées, c'est la légitimité de ce type d'enseignement qui s'en trouve d'une certaine manière établie au sein même de l'université et qui dans le même élan légitime l'évolution de la professionnalité des enseignants chercheurs sur des objets de formation transdisciplinaire. Autant de compétences qu'eux-mêmes avaient acquises ou construites. Jusque là, il leur semblait inapproprié de les transmettre à leurs étudiants dans le cadre d'un enseignement universitaire. Mais cette nouvelle incarnation du métier a pris corps pour eux grâce à la coanimation dans une nouvelle mise en scène de soi comme enseignant.

\section{Références}

Attali A., Bressoux P. (2002) L'évaluation des pratiques éducatives dans les premiers et second degrés. Rapport pour le Haut Conseil de l'Évaluation de l'École. Paris : HCÉÉ.

Barbier J-M. (2005) Voies nouvelles de la professionnalisation. In Sorel M., Wittorski R. (dir.) $L a$ professionnalisation en actes et en questions. Paris : L'Harmattan, 121-139.

Bruner J. (1966). Toward a Theory of Instruction. Harvard : Harvard University Press.

Carnus M-F. (2010) La construction de la professionnalité enseignante à travers un dispositif interdisciplinaire de collège. Revue française de pédagogie, 2010, n¹73, 19-40.

Carré P., Charbonnier O. (2003) Les apprentissages professionnels informels. Paris : L'harmattan.

Cuisinier F., Gay M-C., Jeoffrion C., Schneider B. (2006) Regards actuels des enseignants-chercheurs sur leur métier : enquête auprès des enseignants-chercheurs de psychologie. Bulletin de psychologie, 2006-2 n $482,237-249$.

Erlich V., Verley E. (2010) Une relecture sociologique des parcours des étudiants français : entre segmentation et professionnalisation. Éducation et sociétés, 2010-2 n²6, 71-88.

Fave-Bonnet M-F. (2011) Formation pédagogique et développement professionnel des enseignants du supérieur. Recherche et formation, 68-2011, 127-136.

Forestier G. (2002) Ce que coaching veut dire. Paris : Edition d'Organisation.

Houssaye J. (2000) Le triangle pédagogique. Berne : Peter Lang ( $3^{e}$ éd.).

Kirkpatrick D.L. (1994) Evaluating training programs: The four levels. San Francisco : Berrett-Koehler.

Knight P-T., Tait J., Yorke M. (2006) The professionnal learning of teachers in higher education. Studies in Higher Éducation, 31-3, 319-339.

Langevin L., Bruneau M. (2000) Enseignement supérieur. Vers un nouveau scénario. Issy-lesMoulineaux : ESF éditeur.

Langevin L., Bilodeau H., Boisclair M., Bracco M-A. (2007) Formation et soutien à l'enseignement universitaire : des constats et des exemples pour inspirer l'action. Sainte-Foy : Presses de l'université de Québec. 
Leclercq E. (2009) Quand la lutte contre les inégalités intègre la pédagogie universitaire. In Leclercq E., Niclot D. Former des professionnels de la formation en Europe. Reims : EPURE, 223-245.

Leclercq E. (2012) Enseigner le projet professionnel personnalisé : une nouvelle approche pédagogique pour les enseignants chercheurs ? Carrefours de l'éducation, 2012-2 n³4, 161-175.

Léon A. (1971) Psychopédagogie des adultes, Paris : PUF.

Maingueneau D. (1996) Les termes clés de l'analyse du discours. Paris : Seuil.

Monfort V. (2000) « Normes de travail et réussite scolaire chez les étudiants de première année de sciences ». Sociétés contemporaines, 40, 57-76.

Pastré P. (2008) Apprentissage et activité. In Lenoir Y., Pastré P. (dir.) Didactique professionnelle et didactiques disciplinaires en débat. Toulouse : OCTARES éditions, 53-79.

Paul M. (2004) L'accompagnement : une posture professionnelle spécifique. Paris : L'Harmattan. Savoir et formation.

Perrenoud Ph. (2000) Métier d'élève et sens du travail scolaire. Paris : ESF (4éme éd.).

Perrenoud Ph. (2001) Le travail sur l'habitus dans la formation des enseignants. Analyse des pratiques et prise de conscience. In Paquay L., al. (éds.) Former des enseignants professionnels. Bruxelles : De Boeck Université, 181-207.

Rege Colet N., Berthiaume D. (2009) Savoir ou être ? Savoirs et identités professionnels chez les enseignants universitaires. In Hofstetter R., Schneuwly B. (dir.) Savoirs en (trans)formation. Au cœur des professions de l'enseignement et de la formation. Bruxelles : De Boeck, 137-162.

Robert A-D., Bouillaguet A. (2007) L'analyse de contenu. Paris : PUF.

Romainville M., Donnay J. (dir.) (1996) Enseigner à l'université, un métier qui s'apprend ? Bruxelles : De Boeck.

Stes A., Van Petegem P. (2011) La formation pédagogique des professeurs dans l'enseignement supérieur. Recherche et formation, 67, 15-30.

Trigwell K., Prosser M. (2004) Development and Use of the Approaches of Teaching Inventory. Educational Psychology Review, 16-4, 409-424.

Vasconcellos M (2006) L'enseignement supérieur en France. Paris : La découverte.

Vial M., Caparros-Mencacci N. (2007) L'accompagnement professionnel ? Méthode à l'usage des praticiens exerçant une fonction éducative. Bruxelles : De Boeck Université.

Wittorski R. (2007) Professionnalisation et développement professionnel. Paris : L'Harmattan. 